\title{
Reducing Risk by Reducing Hazard
}

\section{Use of Chemical Hazard Screening as the First Step in the Assessment Process}

\author{
Hans Wendschlag \\ Hewlett-Packard Co. \\ Stockholm, Sweden \\ hans.wendschlag@hp.com \\ +46852494906 \\ Helen Holder \\ Hewlett-Packard Co. \\ Palo Alto, USA
}

\author{
Cory Robertson \\ Hewlett-Packard Co. \\ Boise, USA
}

\author{
Curtis Wray \\ Hewlett-Packard Co. \\ Fort Collins, USA
}

\begin{abstract}
With the introduction of materials specific legislation, such as RoHS in 2003, the pace of change of materials in the electronics industry has seen a dramatic increase. Changing materials is costly, and an important business need is the development of a process to select alternatives that will not need to be substituted in the future. To guard against multiple substitutions, the alternatives selected need to have lower impacts on human health and the environment. According to the principles of green chemistry the most efficient way to reduce overall risk is to use less hazardous materials in product design. To guard against regrettable substitution, and future restriction, an alternatives assessment process including chemical hazard assessment as the first step in the process is described. Example applications of the alternatives assessment process are introduced.
\end{abstract}

\section{INTRODUCTION}

The electronics industry faces increasing regulatory and consumer pressure to remove substances of concern from electronic and electrical products. The pace of change in the materials electronics has increased substantially since the adoption of RoHS in February 2003. EU Directive 2002/95/ $E C$ on the Restriction of the Use of Certain Hazardous Substances in Electrical and Electronic Equipment (commonly referred to as RoHS) was part of an effort to reduce the inherent toxicity of electronics waste and to mitigate the effects of its disposal. The RoHS restrictions on the use of lead, mercury, cadmium, hexavalent chromium, polybrominated biphenyls and polybrominated diphenyl ethers created an instant need for alternative materials.

In order to make an informed decision data on the alternatives is needed. The RoHS restricted substances had been used in electronics for many years and were well studied. Many were concerned that the alternative substances had not been sufficiently evaluated to ensure that they would have less impact on human health and the environment. Assessments showed mixed results for the alternatives in comparison with the original materials [1] [2].

The EU directive does not indicate what the alternatives should be so manufacturers and their suppliers developed their own criteria for selecting alternatives. For some suppliers this is an informal process with cost being the primary criteria, other suppliers have a more formal process with multiple criteria. From a business perspective, it is undesirable to face future restrictions for the same application due to a poor choice of replacement materials. Changing materials only after careful consideration of the alternatives will help avoid unintended consequences and prevent regrettable substitutions [3]. Uninformed decisions may cause manufacturers to change materials multiple times, incurring the cost of transition each time [4]. Chemical substitutions can be costly, and the required changes can be disruptive to product releases. In light of the trend towards more chemical regulation and substance restriction, there is a growing risk of multiple substitutions unless potential replacement technologies are properly assessed against environmental and human health criteria in advance of their widespread adoption.

A comprehensive approach accounting for the many variables of material selection, including environmental and human health concerns, is needed. The emerging field of green chemistry addresses many of the core issues of sustainability at the design phase, where the most efficient change is achieved.

\section{GREEN CHEMISTRY}

The term green chemistry shares similar concepts with other programs such as benign by design, pollution prevention and design for the environment. The definition of green chemistry describes all of these concepts, "Green chemistry is the utilization of a set of principles that reduces or eliminates the use or generation of hazardous substances in the design, manufacture and application of chemical products" [5].

Risk is a function of hazard and exposure. Traditional approaches to reducing risk have focused on reducing exposure using techniques such as personal protective equipment or encapsulation of toxic materials with non-toxic coatings for instance. Many of the large chemical companies spend as much on environmental health and safety to protect their workers from hazardous chemicals as they do on research and development [5]. The green chemistry approach focuses on reducing risk by using materials that are inherently less hazardous. 


\section{THE GREENSCREEN ${ }^{\mathrm{TM}}$ FOR SAFER CHEMICALS}

Recognizing the need for a better way to evaluate whether alternatives have a lower overall adverse impact to human health and the environment, an integrated assessment approach was developed for analyzing potential replacements. This integrated approach incorporates a comparative chemical hazard screening step based on a tool called the GreenScreen $^{\mathrm{TM}}$ for Safer Chemicals, a framework launched in 2007 by the non-governmental organization Clean Production Action [6].

The GreenScreen ${ }^{\mathrm{TM}}$ is an open-source, comparative chemical hazard assessment tool. The tool provides a clear and transparent decision logic that assesses 17 globally harmonized endpoints for environmental fate, human health, and environmental toxicity, and then generates benchmark scores ranging from the most to the least hazardous. The hazard endpoints are shown in Figure 1.

Environmental Fate
- Persistence
- Bioaccumulation
Environmental Health
- Acute Aquatic Toxicity
- Chronic Aquatic Toxicity
Human Health Group I
- Carcinogenicity
- Mutagenicity and Genotoxicity
- Reproductive Toxicity
- Developmental Toxicity
- Endocrine Activity
Human Health Group II
- Acute Mammalian Toxicity
- Systemic Toxicity and Organ Effects
- Neurotoxicity
- Respiratory Sensitization
- Skin Irritation
- Eye Irritation
Physical Hazards
- Reactivity
- Flammability

Figure 1: GreenScreen ${ }^{\mathrm{TM}}$ version 1.2 hazard endpoints and groups

An important attribute of the GreenScreen ${ }^{\mathrm{TM}}$ is the alignment with the Globally Harmonized System of Classification and Labeling of Chemicals (GHS) and with the U.S. EPA Design for Environment (DfE) program's chemical alternatives assessment approach [4]. By aligning with these programs the assessments are more likely to identify chemicals that may be regulated in the future.

Another important feature of the GreenScreen ${ }^{\mathrm{TM}}$ is the simple 1 to 4 benchmark score. The GreenScreen defines four benchmark levels for substances:

Benchmark 1 -“Avoid - Chemical of High Concern"

Benchmark 2 -"Use but Search for Safer Substitutes"

Benchmark 3 -"Use but Still Opportunity for Improvement"

Benchmark 4 -"Prefer - Safer Chemical"

Once an expert assessor evaluates a group of chemicals, the results can easily be translated into procurement guidance for people without toxicology or chemistry backgrounds. The simple integer scoring system streamlines the decision making process putting the results in the hands of decision makers.

Hazard assessments are significantly faster and easier to complete than life cycle assessments or risk assessments because hazard endpoints are well-defined and are based on directly observable intrinsic traits or the behavior of a substance. Also, hazard data are generally available now that new EU chemical regulations require more disclosure of test data $(\mathrm{REACH})$ [7]. The GreenScreen ${ }^{\mathrm{TM}}$ is not a replacement for risk assessment or life cycle assessment but it is very effective when used early in the product design process.

\section{INTEGRATED AlternAtives AsSESSMENT PROTOCOL} (IAAP)

In order to maximize resources and utilize the strengths of the GreenScreen $^{\mathrm{TM}}$, life cycle assessment and risk assessment, HP uses an integrated alternatives assessment protocol (IAAP). The GreenScreen ${ }^{\mathrm{TM}}$ alone does not address the full range of life cycle and exposure impacts and the IAAP provides a path to address these issues before final material selection. The IAAP combines the strengths of each assessment tool and allows each tool to be used as intended and at the appropriate time in the product design process.

Figure 2 shows the IAAP flow chart. The first step, identify substances of concern, may result from restriction of a chemical through regulation or by market pressure or scientific findings even before regulation. Once a target substance has been identified it is important to characterize the end use and function of the material, flame retardant alternatives must provide the same level of fire safety for instance. Step three is identify potential alternatives. This step requires working closely with suppliers to identify materials that could meet the environmental, regulatory and quality requirements. It is important to include many options at this point and not screen out too many options in order to promote innovative solutions including designing out the substance or function.

The fourth step introduces the first screening step, assess chemical hazards, and the GreenScreen ${ }^{\mathrm{TM}}$ is used along with R-phrase and restricted substance list (RSL) screening. It is important to assess the chemical hazard first to screen out alternatives that do not have lower impact on human health and the environment. Alternatives that fail to meet this requirement are placed in the deselected options but may be reconsidered if no viable technical solutions are found in step five.

The fifth step in the IAAP is to assess the technical and economic performance of the alternatives. The alternatives must provide equivalent technical performance. Some products may have specific requirements that must be met while the necessary performance requirements may need to be developed for other products, adding an additional challenge. Determining the right performance requirements is a critical step and gives suppliers are target to find the most costeffective solutions. The exact same performance as the original material may or may not be possible and it is important to understand the actual technical performance needed for the specific application. 


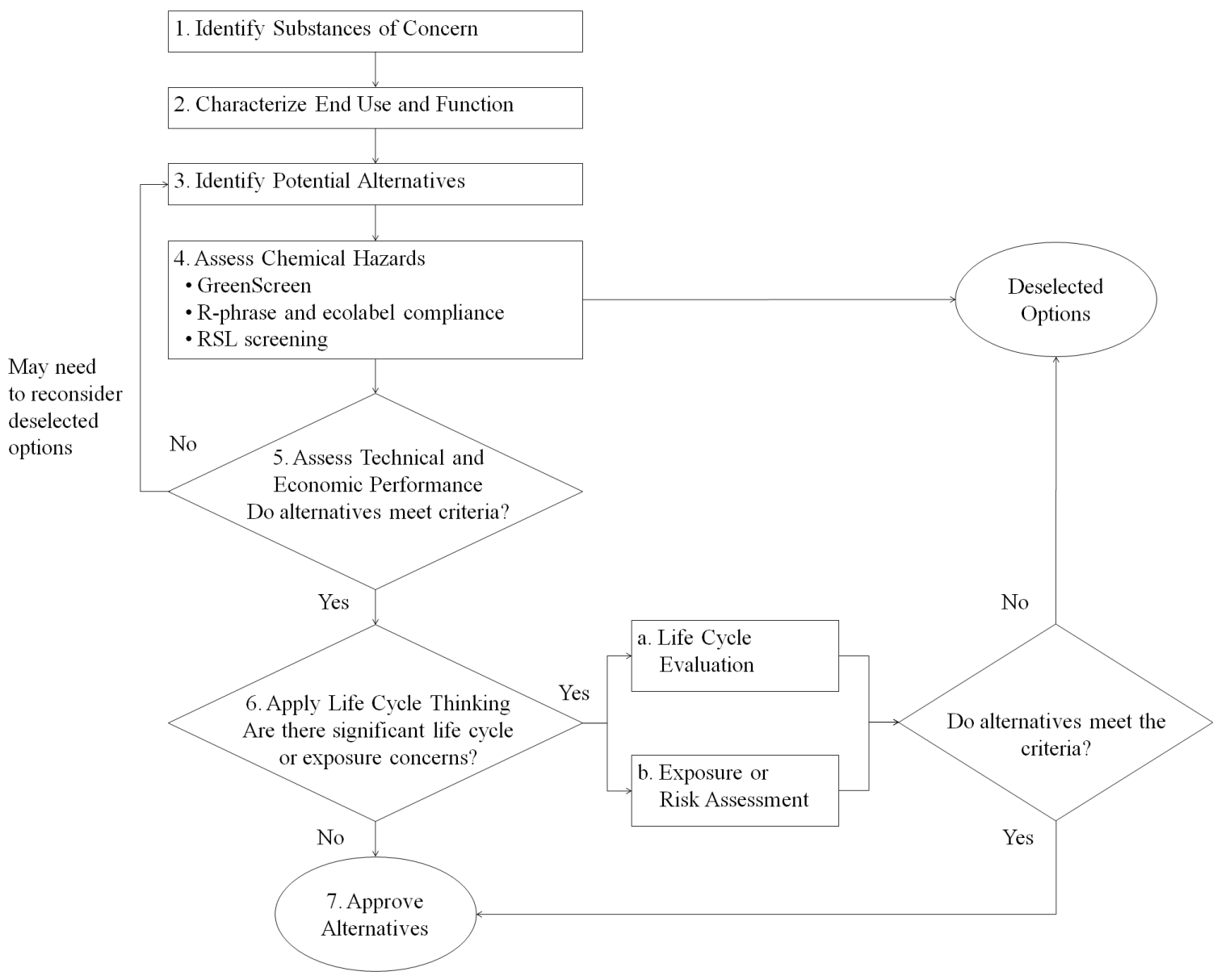

Figure 2. Integrated alternatives assessment protocol (IAAP) for evaluating replacements for restricted substances

Application of life cycle thinking and exposure considerations are considered in step six. A broader range of impacts to human health and the environment are taken into consideration such as global warming, end-of-life disposition and worker exposure. This consideration may be very brief or may trigger exposure studies taking many months to complete depending on the function and alternatives being considered. The TURI Five Chemicals Alternatives Assessment Study provides a good example of life cycle thinking [8].

Finally, in step seven, the alternatives are approved for use in products. The goal of the process is not to find a single optimum alternative but rather to find many acceptable alternatives. A range of acceptable alternatives provides suppliers with flexibility to choose materials that meet the needs of the application. In some instances the list of approved alternatives is documented and becomes a "white list" that can be used to identify the preferred alternatives.

\section{Alternatives Assessment Projects}

Alternatives assessment has been successful in improving materials selection during product development at HewlettPackard using multiple approaches. Alternatives assessment projects range from simple "white lists" to application of the IAAP described previously. Several projects are described below.

\section{A. Flame Retardant and Plasticizer White Lists}

The earliest applications of alternatives assessment included the application of the GreenScreen ${ }^{\mathrm{TM}}$ to a class of materials to identify the preferred alternatives. Flame retardants and plasticizers were the first two classes of materials assessed with the GreenScreen ${ }^{\mathrm{TM}}$. The alternatives were classified into groups based on their benchmark score of $1,2,3$ or 4 . The list can then be used by product designers to quickly associate chemical hazard with a particular alternative.

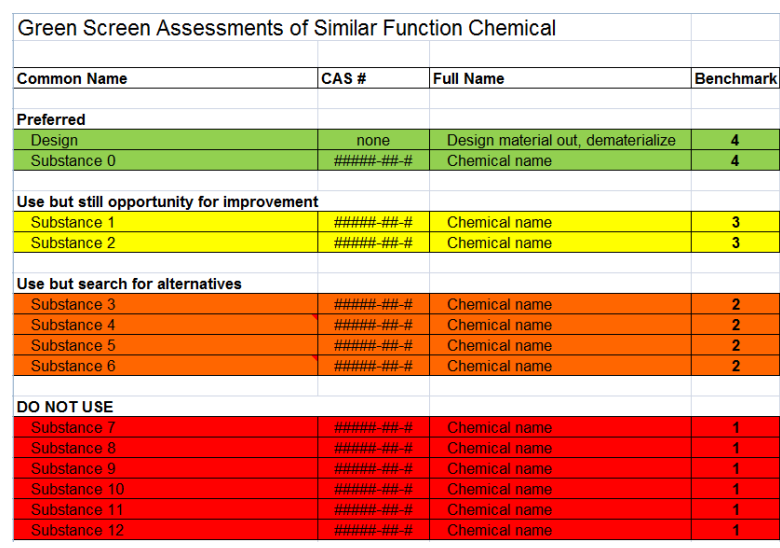

Figure 3: Material Class GreenScreen ${ }^{\mathrm{TM}}$ Summary 


\section{B. PVC-Free Power Cord Project}

PVC is being phased out of many products throughout the electronics industry. In order to prevent regretable substitution, alternatives assessment is a requirement for approval of alternatives to PVC power cords. The IAAP is applied to proposed alternatives and the approved alternatives are placed on an approved materials list. Cable manufacturers can then select materials from this list to construct power cords.

In order to validate the GreenScreen ${ }^{\mathrm{TM}}$ assessments performed by the suppliers or a third party, the formulations of the resins were revealed to HP under a confidentiality agreement. Some materials were deselected based on the GreenScreen ${ }^{\mathrm{TM}}$ results but a number of materials progressed to regulatory testing and have been approved for use. Resin suppliers attended mandatory training on the GreenScreen ${ }^{\mathrm{TM}}$ tool at the HP Fort Collins site in 2010.

Finished power cords were subjected to performance tests and compared to PVC cords as part of IAAP process. The technical requirements, including fire retardancy, were also an important part of the process as the fire safety could not be compromised. Exposure assessments were a consideration in some formulations but for the most part the PVC and alternative cords would be used in the same way and the exposure potential for both is the same. Life cycle thinking was an important consideration, especially at end-of-life due to informal recycling and low temperature incineration.

\section{Cleaners}

$\mathrm{HP}$ is also applying alternative assessment protocol to spot cleaners that are used in the printed circuit board manufacturing process. Cleaners provide a unique case for alternatives assessment in electronics because these chemicals are used primarily in the manufacturing process and are not found in the finished product. Often exposure controls on the manufacturing line are present to reduce risk from cleaning chemicals; nevertheless, exposure controls can fail or may not be sufficient. Furthermore, cleaners used for repair and rework during electronics manufacturing are sometimes less controlled. Often this type of cleaning is done by hand using aerosol application; spot cleaning may also be done by a technician during a field repair. Recent news reports of poisoning from exposure to the cleaning chemicals at manufacturing plants underscore the need to decrease the inherent hazards of cleaners [9].

When we apply the IAAP to common cleaner formulations, many are easily deselected because they are known carcinogens or neurotoxicants. Once these chemicals are precluded, cleaner formulations can be chosen that minimize the hazard for manufacturing workers. Before these less hazardous formulations are adopted, they must meet technical performance criteria. Currently HP is working with cleaner formulators to develop effective cleaners that meet our hazard criteria.

The application of chemical hazard assessment to cleaners has highlighted some shortcomings of our current approach. We must determine a way to adequately assess mixtures of chemicals. For example, the hazard of some cleaner chemicals can be synergistic which must be taken into account during initial assessment [10]. In this case a chemical that may be acceptable by itself may be unacceptable when present with synergists. Conversely, small amounts of hazardous (GreenScreen ${ }^{\mathrm{TM}}$ Benchmark 1) chemicals may be needed to accomplish a particular application. We must determine if there are levels of chemicals that are acceptable to meet performance criteria. This decision can only be made once a thorough alternatives assessment is completed.

\section{Fluxes}

HP is in the initial stages of implementing an alternatives assessment program for solder paste and fluxes. These materials involve potential hazards during manufacturing and at the end-of-life of electronic products. Many flux formulations involve some sort of solvent vehicle and we want to minimize the hazard of these chemicals. Also, many flux residues, like PVC, are concerns due to their halogen content. When electronics are subjected to informal recycling (i.e. open pit burning), the presence of halogens can lead to hazardous byproducts.

\section{CONCLUSIONS}

The GreenScreen ${ }^{\mathrm{TM}}$ uses the principles of green chemistry to provide and important piece of information to product manufacturers, namely a simple benchmark score characterizing chemical hazard in a format that anyone understands. Chemical classification activities today provide a lot of information about chemicals in the marketplace but stop short of ranking chemicals based on their intrinsic hazard. The GreenScreen $^{\mathrm{TM}}$ can provide this missing piece of information that can then be used by decision makers without toxicology background to make meaningful comparisons between material alternatives.

The GreenScreen ${ }^{\mathrm{TM}}$ has been used effectively in multiple projects within HP for several years. As a next step HP has been working with Clean Production Action and various ecolabel organizations to incorporate the GreenScreen ${ }^{\mathrm{TM}}$ as the materials requirement. Ecolabels such as TCO, Nordic Swan, Blue Angel and EPEAT, among others, currently depend on risk phrases and hazard statements for materials requirements which has several drawbacks. Using risk phrases rewards lack of data, if there is no data no risk phrase is assigned. Targeting risk phrases in a certain area of concern may neglect other areas of concern resulting in regrettable substitution. The GreenScreen ${ }^{\mathrm{TM}}$ has minimum data requirements and takes a more comprehensive approach assessing both environmental and human health concerns.

Furthermore, the scoring system allows manufacturers, and potentially ecolabel organizations, to effectively communicate materials goals to suppliers. By requiring a minimum GreenScreen $^{\mathrm{TM}}$ benchmark rating, manufacturers can influence materials formulations at the most basic level. In addition, chemical suppliers could promote high benchmark solutions to product manufacturers. By promoting the use of benign materials up the supply chain where formulation decisions are made, the GreenScreen ${ }^{\mathrm{TM}}$ drives change where it is most effective: at the molecular level.

\section{REFERENCES}

[1] C.A. Ascencio, J.J. Madsen, "LCA comparison of alternative soldering techniques," PRé Consultants bv, 2005.

[2] J. R. Geibig, "Solders in Electronics: A Life-Cycle Assessment,“" EPA 744-R-05-001, 2005. 
[3] R. D. McFadden, "The business case for transitioning to safer chemicals," New Solutions 21, no. 3:403-416, 2011.

[4] E. T. Lavoie, G. Heine, H. Holder, M. S. Rossi, R. E. Lee, E. A. Conner, M. A. Vrabel, D. M. Difiore and C. L. Davies. "Chemical alternatives assessment: Enabling substitution to safer chemicals." Environmental Science and Technology 44 (24): 9244-9249, 2010.

[5] P. T. Anastas and J. C. Warner. "Green chemistry: Theory and practice,“New York: Oxford University Press Inc., 1998, p. 11.

[6] Clean Production Action, "GreenScreen ${ }^{\mathrm{TM}}$ for Safer Chemicals," [Online] Available: http:// www.cleanproduction.org/greenscreen.php

[7] "EC Regulation on Registration, Evaluation, Authorisation and Restriction of Chemicals“(REACH), No 1907/2006, 2007.

[8] M. Ellenbecker, "Five Chemicals Alternatives Assessment Study,“ Toxics Use Reduction Institute, University of Massachusetts, Lowell, 2006.

[9] D. Barboza, "Workers sickened at Apple supplier in China," [Online] Available: www.nytimes.com/2011/02/23/ technology/23apple.html

[10] O. Ladefoged, U. Hass and L. Simonsen, "Neurophysiological and behavioural effects of combined exposure to 2,5hexanedione and acetone or ethanol in rats," Pharmacol. Toxicol. 65:372-375, 1989. 